\title{
Una mirada general a las enfermedades pulmonares intersticiales y una específica a la fibrosis pulmonar idiopática
}

\author{
A general overview of interstitial lung diseases \\ with special focus on idiopathic pulmonary fibrosis
}

Pedro J Marcos, Carmen Montero, Isabel Otero González

Servicio de Neumología. Instituto de Investigación Biomédica de A Coruña. Xerencia de Xestión Integrada de A Coruña. SERGAS. A Coruña

\section{Introducción}

Las enfermedades pulmonares intersticiales difusas (EPID) engloban un conjunto heterogéneo de patologías caracterizadas por afectar predominantemente al intersticio pulmonar, que es el espacio anatómico comprendido entre las membranas basales del epitelio alveolar y el endotelio capi$\operatorname{lar}^{1}$. Con frecuencia se observa un retraso en el diagnóstico de las EPID. La falta de especificidad de los síntomas y el escaso uso de técnicas diagnósticas como es la espirometría en los primeros niveles asistenciales, hacen que sea bastante frecuente que los clínicos achaquen los síntomas respiratorios del paciente a entidades más prevalentes como es la enfermedad pulmonar obstructiva crónica (EPOC).

La evaluación diagnóstica de las EPID comienza con un abordaje estructurado con una extensa historia clínica, detenida exploración física y una meticulosa historia ocupacional, exposicional y farmacológica.

Con esta revisión queremos hacer una aproximación general al abordaje de las EPID, destacando finalmente las características fundamentales de la fibrosis pulmonar idiopática (FPI), dentro de las EPID una de las más frecuentes, de etio- logía desconocida, muy mal pronóstico y escasos recursos terapéuticos.

\section{Definición}

Las EPID constituyen un grupo heterogéneo de procesos que se van a caracterizar en ocasiones por la infiltración celular de las estructuras alveolo-intersticiales del pulmón, aunque no exclusivamente. En ocasiones pueden afectan las pequeñas vías respiratorias y la vasculatura pulmonar.

Existen más de 150 entidades que puedan provocar EPID y suelen compartir características clínicas, radiológicas o funcionales ${ }^{1}$. Cuando estas entidades no se resuelven espontáneamente 0 tras un tratamiento específico conducen a una fibrosis pulmonar, en donde las unidades alveolo-capilares han sido destruidas y sustituidas por tejido fibroso y en donde existe una grave alteración del intercambio gaseoso.

\section{Clasificación}

En el momento actual la clasificación más aceptada y empleada universalmente es la realizada por consenso entre la American Thoracic Society (ATS) y la European Respiratory Society (ERS) en el año 2002 (Figura 1)². Esta clasificación

Figura 1. Clasificación de las enfermedades pulmonares intersticiales difusas (EPID)

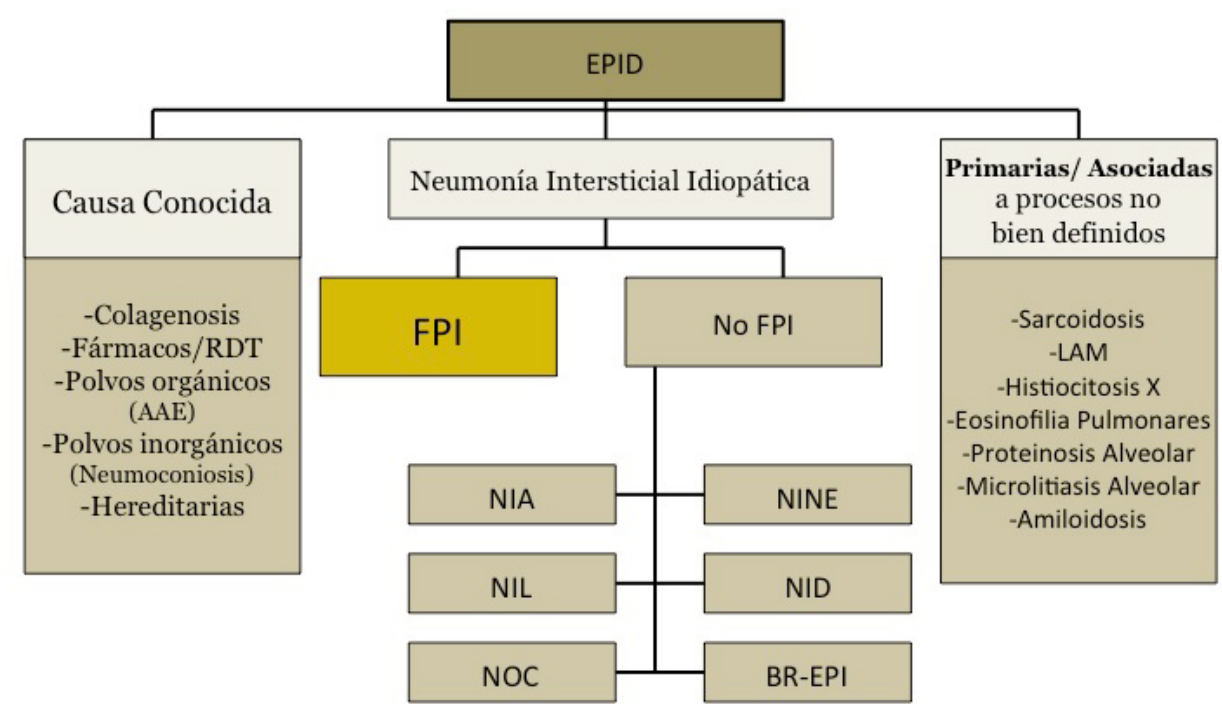

BR-EPID: bronquiolitis respiratoria asociada a EPID; FPI: fibrosis pulmonar idiopática; NIA: neumonía intersticial aguda; NID: neumonía intersticial descamativa; NII: neumonías intersticiales idiopáticas; NIL: neumonía intersticial linfoide; NINE: neumonía intersticial no específica; NOC: neumonía organizada criptogénica. RDT: radioterapia. AAE: alveolitis alérgica extrínseca. LAM: linfangioleiomiomatosis. 

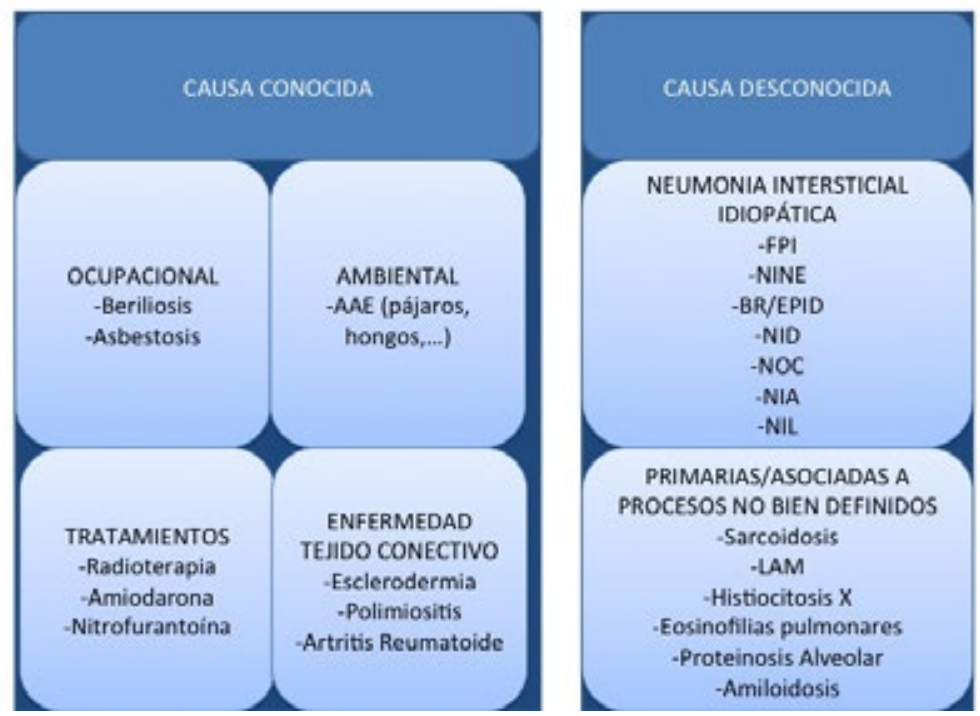

concede bastante importancia a la histopatología, por lo que quizás de cara a la práctica clínica lo más práctico es dividir las entidades en función de que su causa sea conocida 0 no (Figura 2). La revisión de exposiciones ambientales, farmacológicas, ocupacionales o de hobbies, así como la búsqueda sistemática de síntomas y signos sugestivos de enfermedades del tejido conectivo puede ayudar al clínico a acercarse al diagnóstico y en ocasiones al pronóstico de la enfermedad. Generalmente, las formas no idiopáticas tienden a tener un mejor pronóstico y responder al tratamiento. Así, por ejemplo, la detección de una EPID en relación con la toma de un fármaco, puede, en muchos casos, resolverse simplemente tras la retirada del mismo.

Las EPID sin causa conocida abarcan un abanico amplio que va desde las enfermedades granulomatosas como la sarcoidosis a las neumonías intersticiales idiopáticas (NII), dentro de las cuales se encuentra la fibrosis pulmonar idiopática (FPI), la más frecuente de ellas y de las de peor pronóstico. En muchas ocasiones, la detección de fibrosis pulmonar es el resultado de la evolución en el tiempo de una patología intersticial de la que va a resultar casi imposible reconocer su causa inicial.

A pesar del elevado numero de procesos causales de EPID la diferenciación de sus distintas formas es fundamental dada la gran variabilidad que existe en cuanto a la respuesta al tratamiento y el pronóstico. Así mientras que los esteroides e inmunosupresores pueden ser eficaces en el tratamiento de patologías como la sarcoidosis, no existe ninguna terapia eficaz para el tratamiento de la FPI. Esta entidad, con una mediana de supervivencia comprendida entre los 3 y los 5 años, solo tiene, y en casos seleccionados, el trasplante pulmonar como tabla de salvación.

\section{Epidemiología}

Se estima que un $15 \%$ de la patología respiratoria atendida está relacionada con las EPID ${ }^{3}$. Los estudios epidemiológicos en este campo son escasos, los cambios realizados en su clasificación y que la mayoría de los registros de EPID se han elaborado mediante encuestas dirigidas a servicios de neumología. Un estudio clásico realizado en Nuevo México y publicado en 1994 encontró una prevalencia de 80.9 casos por 100.000 habitantes en hombres y 67.2 por 100.000 habitantes en mujeres ${ }^{4}$.

Por orden de frecuencia las EPID más prevalentes son la FPI y la sarcoidosis, seguidas por las alveolitis alérgicas extrínsecas y las asociadas a las enfermedades del colágeno $0^{5,6}$.

\section{Diagnóstico}

\section{Situaciones de sospecha}

La ausencia de sintomatología específica puede ocasionar que en fumadores, con enfermedad incipiente el cuadro pase desapercibido y sea atribuido a la EPOC. La disnea progresiva o la persistencia de la tos, incluso después de haber dejado de fumar o la falta de respuesta a un tratamiento empírico con broncodilatadores pueden sugerir que estamos ante una EPID.

La exploración física puede ser normal, la ausencia de sibilantes, la presencia de crepitantes, que suelen ser secos y en las bases pulmonares, y la presencia de acropaquias pueden sugerir al clínico una EPID.

Desde el punto de vista radiológico, la presencia de las más sutiles imágenes intersticiales o nodulares 0 la ausencia de mejoría radiológica tras un ciclo de antibióticos deberían hacernos sospechar que nos encontramos ante una EPID.

Por último, mientras que el patrón espirométrico de la EPOC es el obstructivo, en las EPID, en su gran mayoría es el restrictivo.

\section{Anamnesis}

La realización de una completa historia clínica es fundamental para un correcto abordaje de estas patologías.

Habrá que tener en cuenta la edad del paciente, sexo e historia familiar de enfermedad. También los antecedentes exposicionales tanto laborales, ocupacionales como hobbies. 
La anamnesis laboral debe ser extensa e incluir las distintas actividades laborales realizadas en orden cronológico, fechas en las que tuvieron lugar las exposiciones de riesgo y la duración de las mismas. Los tratamientos farmacológicos recibidos no son causa infrecuente de EPID, por lo que deberán anotarse todos los que toma 0 ha tomado, la dosis y duración del mismo. La radioterapia, fundamentalmente la torácica puede ser causa de EPID y habrá que tenerla en consideración en el diagnóstico diferencial.

Por último, no debemos olvidar que la EPID puede ser la primera manifestación de una enfermedad sistémica, por lo que será importante indagar sobre cualquier sintomatología extrapulmonar.

\section{Clínica}

Los síntomas son muy inespecíficos. El más frecuente es la disnea, habitualmente con un perfil de inicio lentamente progresivo y durante un tiempo puede ser el único síntoma. La tos, con frecuencia improductiva, es otro síntoma princeps. La aparición de forma más aguda o subaguda de fiebre y otros síntomas sistémicos pueden hacernos orientar a la presencia de neumonía intersticial aguda (NIA), alveolitis alérgicas extrínsecas (AAE), neumonitis por fármacos, neumonía organizada criptogénica (NOC) y eosinofilias pulmonares. La hemoptisis es poco frecuente aunque puede aparecer en pacientes con linfangioleiomiomatosis (LAM). El dolor torácico también es infrecuente y, cuando es pleurítico, podría ser el reflejo de la aparición de un neumotórax y orientar al diagnostico de una LAM o Histiocitosis $X^{1,7}$.

En ocasiones la enfermedad pude asociar síntomas o signos extrapulmonares que pueden estar presentes en diversas enfermedades asociadas a las EPID (Tabla 1).

\section{Exploración Física}

La mayoría de los pacientes no tienen sibilantes en la auscultación (con excepción de las eosinofilias pulmonares o las AAE) un signo que indica enfermedad de vía aérea. Sin embargo tendrán con frecuencia crepitantes inspiratorios, sonido que representa la apertura de los alveolos colapsados contra un espacio intersticial fibrosado que los rodea. La presencia de acropaquias es relativamente frecuente en la FPI.

Existen otros datos exploratorios que pueden orientar a determinadas patologías. Así la detección de adenopatías, eritema nodoso o lupus pernio es característico de la sarcoidosis. Las artralgias son frecuentes en las EPID, pero una sinovitis activa sugerirá una enfermedad del tejido conectivo (ETC). Los pacientes con esclerodermia pueden tener esclerodactilia, fenómeno de Raynaud o lesiones telangiectásicas. La debilidad en cinturas, la presencia de las llamadas manos de mecánico o lesiones cutáneas como las pápulas de Gottron o el eritema heliotropo indicarían la presencia de una polimiositis/dermatomiositis.

\section{Hallazgos analíticos}

El principal beneficio de la realización analítica sanguínea es la
Tabla 1. Afectación sistémica en las EPID

\section{Sistema nervioso}

${ }^{*}$ Central: sarcoidosis, neurofibromatosis, esclerosis tuberosa, ETC

*Periférico: ETC

Ojos

*Escleritis: lupus, esclerodermia, sarcoidosis

*Queratoconjuntivitis seca: síndrome de Sjögren

*Uveítis: sarcoidosis

*Aumento de la glándula lacrimal: sarcoidosis

Aparato musculosquelético

*Miositis: enfermedades del colágeno

*Artritis: sarcoidosis, ETC

*Afectación ósea: Histiocitosis X, sarcoidosis

Riñón

*Angiolipomas: linfangioleiomiomatosis

*Síndrome nefrótico: amiloidosis, lupus

*Glomerulonefritis: ETC

\section{Corazón}

*Miocardio: sarcoidosis

*Pericardio: ETC

Aparato digestivo

*Hepatosplenomegalia: sarcoidosis, histiocitosis X, ETC, amiloidosis

*Diarrea crónica: enfermedad inflamatoria intestinal

*Disfagia: esclerosis sistémica, dermatomiositis/polimiositis Piel

*Eritema nodoso: sarcoidosis, enfermedades del colágeno

*Nódulos subcutáneos: artritis reumatoide, neurofibromatosis, sarcoidosis

*Neurofibromas y manchas "café con leche": neurofibromatosis

*Exantema heliotropo: dermatomiositis

*Albinismo: síndrome de Hermansky-Pudlak

\section{Metabólico}

*Diabetes insípida: sarcoidosis, histiocitosis X

*Hipercalcemia: sarcoidosis

\section{ETC: enfermedad del tejido conectivo}

demostración de afectación sistémica, la detección de ETC y el establecimiento de un potencial antígeno causal en la AAE.

Las determinaciones realizadas con más frecuencia en el diagnóstico de la EPID, y alguno de los diagnósticos que pueden sugerir sus resultados se pueden ver en la Tabla 2.

Puede existir una elevación de los reactantes de fase aguda, como la proteína $C$ reactiva, la lactatodeshidrogenasa (LDH) y la velocidad de sedimentación globular (VSG). Los anticuerpos antinucleares (ANA) y el factor reumatoide son positivos en el $10-20 \%$ de los pacientes con EPID, aunque a títulos bajos. La presencia de ANA con una titulación elevada (>1:160) debe sugerir una enfermedad del tejido conectivo. La enzima de conversión de la angiotensina está ocasionalmente elevada en la sarcoidosis. No tiene valor diagnóstico, puede elevarse en otras EPID y su utilidad como forma de valorar la actividad de la sarcoidosis es controvertida ${ }^{8}$.

Los estudios analíticos de la neumonitis por hipersensibilidad deben ir dirigidos a la determinación de los anticuerpos IgG. Los falsos positivos y negativos son frecuentes por lo 
Tabla 2. Determinaciones analíticas y patologías de sospecha en las EPID

\begin{tabular}{|l|l|}
\hline Determinación & Patologia de sospecha \\
\hline Hemograma & $\begin{array}{l}\text { Eosinofilia: Neumonía eosinofílica aguda } \\
\text { y crónica }\end{array}$ \\
\hline Urea y creatinina & \\
\hline Electrolitos & $\begin{array}{l}\text { Hipercalcemia, hipercalciuria: } \\
\text { sarcoidosis }\end{array}$ \\
\hline Calcio y fósforo & \\
\hline VSG & \\
\hline PCR & \\
\hline LDH & \\
\hline ANA & LES \\
\hline Anti Ds-DNA(SLE) & LES \\
\hline Anti-Smith & Artritis reumatoide \\
\hline Factor Reumatoide & Artritis reumatoide \\
\hline $\begin{array}{l}\text { Anticuerpos anti Péptido } \\
\text { cíclico citrulinado }\end{array}$ & Sjogren's \\
\hline Anti-SSA/Ro & Sjogren's \\
\hline Anti-SSB/La & Polimiositis/Dermatomiositis \\
\hline CPK y Aldolasa & Polimiositis/Dermatomiositis \\
\hline Anti Jo1 & EMTC \\
\hline Anti RNP & Esclerodermia \\
\hline Anti Scl-70 & Poliangeítis microscópica \\
\hline ANCA-p & Wegener \\
\hline ANCA-c & Alveolitis alérgica extrínseca \\
\hline Precipitinas & Serteros stemico \\
\hline
\end{tabular}

LES: lupus eritematoso sistémico.

EMTC: enfermedad mixta del tejido conectivo.

que hay que interpretar estos datos con cautela. Un resultado positivo puede ser simplemente un marcador de exposición y un falso negativo puede estar relacionado con haber testado el antígeno erróneo o haber cesado la exposición. Dada la alta tasa de falsos negativos y positivos tanto en las ETC como en la neumonitis por hipersensibilidad, los hallazgos analíticos no debieran suplantar al diagnóstico clínico.

\section{Radiología}

La radiografía simple de tórax es la exploración radiológica fundamental en la primera aproximación de una EPID porque es capaz de detectar a la gran mayoría de pacientes, permite hacer una aproximación diagnóstica en función del patrón detectado y su comparación en el tiempo y de manera seriada es útil para el seguimiento de la enfermedad.

La tomografía computarizada (TC) torácica es más sensible y más específica que la radiografía simple para el estudio de las alteraciones del intersticio pulmonar. En el estudio de las EPID debe realizarse siempre la TC de alta resolución (TCAR).

La TCAR también nos ayuda en la estimación de la reversibilidad y el pronóstico de la enfermedad difusa. En general, las imágenes en vidrio deslustrado son indicativas de inflamación y potencialmente reversibles, mientras que las imágenes de reticulación y la panalización indican fibrosis y cambios irreversibles.
Mediante el TCAR es posible determinar el patrón radiológico más característico (reticular, consolidación, vidrio deslustrado, nódulos), distribución anatómica (superior/ inferior; central/periférica) y otros hallazgos añadidos (presencia de adenopatías, atenuación en mosaico, empedrado, derrame pleural...). La administración de contraste con el TC generalmente no es necesaria, pero podría en algunos casos, fundamentalmente en sarcoidosis, ayudar a la hora de diferencia adenopatías hilio-mediastínicas de estructuras vasculares. En la Tabla 3 podemos ver distintos patrones radiológicos con sus correlaciones clínicas más frecuentes. Así mismo la TCAR servirá de ayuda a la hora de seleccionar el lugar adecuado para la práctica del lavado broncoalveolar (LBA) y de las biopsias sean transbronquiales o quirúrgicas ${ }^{9}$.

\section{Estudio funcional respiratorio}

Ante todo paciente con síntomas respiratorios crónicos está indicada la realización de una espirometría. El patrón espirométrico característico es el llamado patrón restrictivo, caracterizado por una reducción de la capacidad vital forzada (FVC) y un cociente entre el volumen espirado en el primer segundo (FEV1) y la FVC (FEV1/FVC) mayor de 70. Los pacientes con EPOC, pueden tener una FVC disminuida

Tabla 3. Principales patrones radiológicos y su distribución en las EPID.

\begin{tabular}{|c|c|c|}
\hline Diagnóstico & Patrón TCAR & Distribución TCAR \\
\hline $\begin{array}{l}\text { Fibrosis } \\
\text { Pulmonar } \\
\text { Idiopática }\end{array}$ & $\begin{array}{l}\text { Reticular } \\
\text { Panalización } \\
\text { Bronquiectasias de } \\
\text { tracción }\end{array}$ & $\begin{array}{l}\text { Periférica } \\
\text { Subpleural } \\
\text { Basal }\end{array}$ \\
\hline $\begin{array}{l}\text { Neunomía } \\
\text { Intersticial No } \\
\text { Específica }\end{array}$ & $\begin{array}{l}\text { Reticular } \\
\text { Vidrio deslustrado }\end{array}$ & $\begin{array}{l}\text { Periférica } \\
\text { Basal }\end{array}$ \\
\hline $\begin{array}{l}\text { Neumonía } \\
\text { Organizada } \\
\text { Criptogégica } \\
\end{array}$ & Consolidación & $\begin{array}{l}\text { Periférica } \\
\text { Peribronquial }\end{array}$ \\
\hline $\begin{array}{l}\text { Neumonía } \\
\text { Intersticial Aguda }\end{array}$ & $\begin{array}{l}\text { Consolidación } \\
\text { Vidrio deslustrado }\end{array}$ & Difusa \\
\hline $\begin{array}{l}\text { Neumonía } \\
\text { Intersticial } \\
\text { Descamativa } \\
\end{array}$ & $\begin{array}{l}\text { Vidrio deslustrado } \\
\text { Retículo }\end{array}$ & Lóbulos inferiores \\
\hline $\begin{array}{l}\text { Neumonitis por } \\
\text { Hipersensibilidad }\end{array}$ & $\begin{array}{l}\text { Vidrio deslustrado } \\
\text { Nódulos } \\
\text { centrilobulillares } \\
\text { Mosaico }\end{array}$ & Lóbulos superiores y medios \\
\hline Sarcoidosis & $\begin{array}{l}\text { Adenopatías } \\
\text { Opacidades } \\
\text { nodulares } \\
\text { Perilinfático }\end{array}$ & Lóbulos superiores y medios \\
\hline LAM & Quistes & Panpulmonar \\
\hline Histiocitosis X & Quistes & $\begin{array}{l}\text { Lóbulos superiores y medios } \\
\text { Respeta bases }\end{array}$ \\
\hline Silicosis & $\begin{array}{l}\text { Adenopatías } \\
\text { calcificadas } \\
\text { Nódulos } \\
\end{array}$ & Lóbulos superiores y medios \\
\hline Asbestosis & $\begin{array}{l}\text { Reticular } \\
\text { Panalización } \\
\text { Bronquiectasias de } \\
\text { tracción } \\
\text { Placas Pleurales }\end{array}$ & Lóbulos inferiores \\
\hline
\end{tabular}


en relación con el atrapamiento aéreo que en ocasiones padecen, sin embargo en estos casos el patrón predominante es el obstructivo, definido por un cociente FEV1/FVC menor de 7010,11. Algunas enfermedades intersticiales como son la sarcoidosis, histiocitosis $X$, LAM, AAE o neumonía eosinófila son la excepción a la regla general y pueden cursar con un patrón espirométrico obstructivo. La confirmación de un defecto restrictivo será necesario mediante un estudio de los volúmenes pulmonares totales que mostraría una disminución de la capacidad pulmonar total.

Otra característica funcional que destaca en las EPID es la disminución de la capacidad de transferencia pulmonar del CO (DLCO) que es uno de los marcadores funcionales más sensibles de EPID ${ }^{11}$. En los casos en los que coexiste fibrosis pulmonar con enfisema la FVC y los volúmenes pulmonares son normales pero suelen tener una importante disminución de la DLCO.

La gasometría arterial puede mostrar un aumento del gradiente alveolo arterial de oxígeno y ligera hipocapnia. El test de paseo de 6 minutos, es una prueba que cada vez se realiza con más frecuencia ${ }^{12}$. Característicamente los pacientes con EPID presentan disminución de la saturación de oxígeno con el esfuerzo, siendo esta mayor y más precoz en relación con la gravedad de la EPID y el desarrollo de hipertensión arterial pulmonar (HTAP); por otro lado, cada vez hay más trabajos que muestran su utilidad a la hora de evaluar la evolución de la enfermedad.

Los estudios funcionales, principalmente la espirometría además de ser un elemento básico para el diagnóstico, servirá para ver la evolución, orientar el pronóstico y monitorizar la respuesta al tratamiento.

\section{Técnicas broncoscópicas}

La broncoscopia permite obtener muestras citológicas del espacio alveolar (lavado broncoalveolar-LBA) y muestras histológicas del parénquima pulmonar (biopsia transbronquial-BTB). El principal papel de las técnicas endoscópicas en las EPID están el campo del diagnóstico. No se ha demostrado que la práctica seriada del LBA o de la BTB tenga interés en la valoración del pronóstico o de la respuesta al tratamiento de las EPID.

\section{Lavado Broncoalveolar}

El análisis celular e inmunocitoquímico del LBA es de gran interés en la valoración diagnóstica de las EPID. En algunas EPID los hallazgos del LBA pueden tener el peso suficiente que nos permita realizar un diagnóstico de certeza y evitar la realización de una biopsia pulmonar (Proteinosis alveolar, Histiocitosis X, Eosinofilia pulmonar). Sin embargo, generalmente los resultados del análisis del BAL tendrán un valor orientativo, servirá para descartar entidades, fundamentalmente infección y neoplasia, y apoyar diagnósticos (AAE, sarcoidosis....) $)^{1,9}$. Un predominio de linfocitos, aunque puede observarse en la fibrosis pulmonar idiopática, debe ser un motivo para reconsiderar otros diagnósticos, fundamental- mente sarcoidosis en fase de fibrosis o alveolitis alérgica extrínseca $^{13}$. El estudio de poblaciones linfocitarias también tiene interés en algunas entidades como sarcoidosis y AAE. La determinación en el BAL de cuerpos ferruginosos también podría tener su valor a la hora del diagnóstico de patología ocupacional, principalmente la asbestosis ${ }^{14}$.

\section{Biopsia transbronquial}

La biopsia transbronquial (BTB) es una técnica que consiste en obtener una muestra del parénquima pulmonar mediante una pinza introducida a través del broncoscopio. Es relativamente segura en manos expertas pero tiene una serie de contraindicaciones y limitaciones para su aplicación. Así la decisión de realizar una BTB, en ausencia de un diagnóstico clínico-radiológico de certeza, depende de las características del paciente (edad, estado funcional) y de la sospecha diagnóstica. El rendimiento diagnóstico de la BTB dependerá de la distribución topográfica y de la especificidad morfológica de la lesión ${ }^{1,15}$. La BTB tiene una alta rentabilidad en el diagnóstico de sarcoidosis, linfangitis carcinomatosa, daño alveolar difuso, algunas infecciones, proteinosis alveolar y neumonía eosinofílica; rentabilidad moderada en algunas vasculitis, amiloidosis, histiocitosis X o LAM y una baja rentabilidad en el diagnóstico de las NII (con excepción de la NOC), la bronquiolitis constrictiva o enfermedad pulmonar veno oclusiva ${ }^{1,16}$.

Debido a que la variabilidad en el rendimiento diagnóstico de la BTB se debe al pequeño tamaño de las biopsias obtenidas, se ha comenzado a utilizar criosondas, en lugar de la pinza, para la realización de BTB. Aunque es una técnica nueva, parece que el volumen de tejido obtenido es mayor y también su rentabilidad diagnóstica ${ }^{17,18}$. El procedimiento de obtención es más complejo ya que precisa la intubación y sedación profunda del enfermo y existen dudas en cuanto a su nivel de complicaciones. Otra pregunta sobre este tipo de biopsia es la validación de la muestra con respecto a la biopsia quirúrgica en enfermedades con lesiones de predominio subpleural. La biopsia quirúrgica toma muestras muy periféricas y permite el estudio del parénquima en relación con la pleura, mientras que la criobiopsia toma muestras de predominio peribronquiolar. Esperemos en un futuro ver dónde se posiciona en el manejo de las EPID.

\section{Biopsia pulmonar}

En aquellas ocasiones en las que no se haya llegado a un diagnóstico tras una adecuada historia clínica, evaluación del TCAR o bien tras la realización de técnicas endoscópicas estaría indicada la realización de una biopsia pulmonar. Su indicación debe valorarse en cada caso en particular, ya que dependerá del estado clínico del paciente y de las ventajas que pueda implicar desde el punto de vista diagnóstico y terapéutico. El procedimiento más aceptado a día de hoy son las biopsias por videotoracoscopia. La videotoracoscopia supone un menor tiempo operatorio, menos incidencia de complicaciones postoperatorias y reducción de la estancia hospitalaria. Debe existir una buena comunicación entre el 
clínico y el cirujano, junto con el apoyo de las imágenes del TCAR, para decidir las áreas del pulmón en las que se esperaría obtener mayor rentabilidad diagnóstica. Deben tomarse muestras de al menos dos lóbulos y áreas diferentes, una con aspecto macroscópico patológico, y otra con aspecto macroscópico normal. La baja tasa de mortalidad y complicaciones, los abordajes mínimamente invasivos por puerto único y la posibilidad de aplicarse en régimen ambulatorio han facilitado la realización de estos procedimientos ${ }^{19,20}$.

Los resultados de la biopsia pulmonar no deben ser tomados como absolutos a la hora de establecer un diagnóstico. Así determinadas EPID secundarias a exposiciones ocupacionales 0 ambientales se han asociado a patrones histológicos que son completamente indistinguibles de las formas idiopáticas de las EPID. De esta manera, por ejemplo, un trabajador con exposición a asbesto puede desarrollar un cuadro histopatológico pulmonar indistinguible de una FPI o un paciente con exposición al berilio tener un síndrome idéntico a una sarcoidosis. Otro de los problemas de las biopsias pulmonares es la gran variación interobservador existente, fundamentalmente en el diagnóstico de los procesos menos frecuentes ${ }^{7}$. Para complicarlo más, las biopsias de distintos lóbulos de un mismo paciente pueden revelar distintas histopatologías. Esto ocurre de modo característico en pacientes con evidencia de neumonía intersticial no específica en un lóbulo y hallazgos compatibles con FPI en otro; sin embargo en estos casos el prónostico del paciente será similar al visto en los pacientes con FPI?

\section{Algoritmo Diagnóstico}

El proceso diagnóstico de las EPID se caracteriza por ser secuencial, dinámico y multidisciplinar. Secuencial pues la sospecha diagnóstica se establece después de la anamnesis y exploración física e irá variando o reorientándose en función de los hallazgos que tengamos en las distintas exploraciones realizadas. Dinámico puesto que en ocasiones

Figura 3. Pauta diagnóstica de las enfermedades pulmonares intersticiales difusas. (modificado de $\mathrm{e}^{1,12,35}$ )

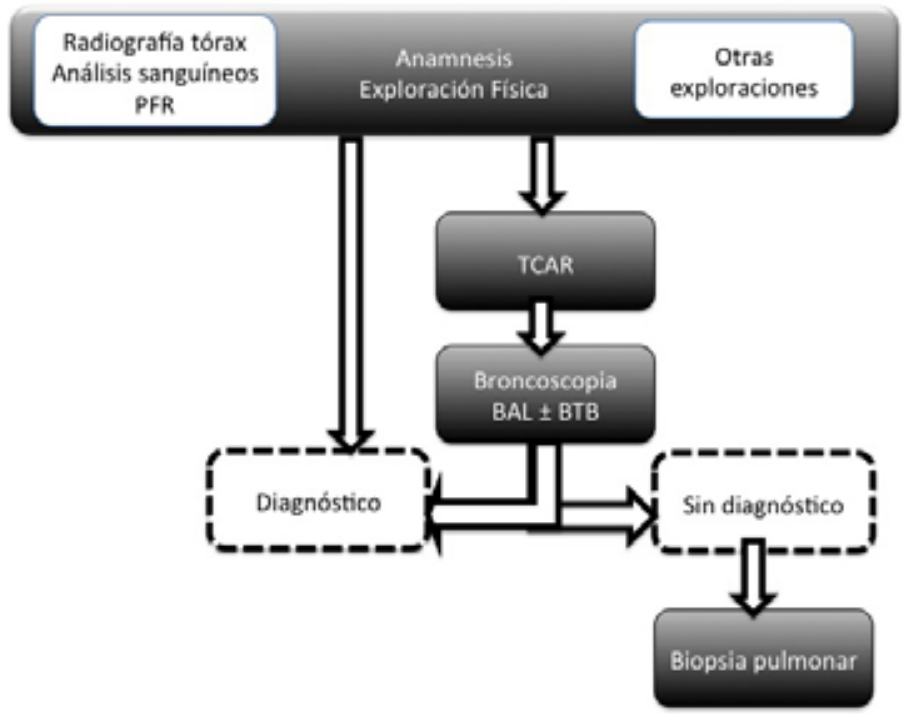

la evolución clínica, funcional o la respuesta al tratamiento pueden llevar a un replanteamiento y cambio del diagnóstico inicial. Finalmente, si algo ha quedado claro en los últimos años, es que el diagnóstico debe ser multidisciplinar. Una valoración multidisciplinar en la que participen neumólogos, radiólogos y patólogos expertos en el diagnóstico y manejo de las EPID consigue aumentar la precisión diagnóstica, siendo en el momento actual el "gold standard" a la hora de establecer un diagnóstico. La aproximación al diagnóstico la podemos observar en la figura 3.

\section{Tratamiento}

A la hora de plantearse el tratamiento de una EPID, deberemos tener en cuenta:

a) evitar la exposición al agente causal, en caso de que exista,

b) suprimir el componente inflamatorio de la enfermedad (alveolitis) cuando esté presente,

c) tratar los síntomas, principalmente la tos y la disnea,

d) evitar las causas agravantes de la enfermedad (reflujo gastroesofágico, infecciones respiratorias, hipertensión pulmonar...),

e) tener en cuenta y tratar los efectos secundarios derivados del tratamiento,

f) el trasplante pulmonar puede ser una alternativa en enfermedad avanzada, siempre y cuando no existan contraindicaciones.

g) ofrecer terapia paliativa en la fase final de la enfermedad.

El primer objetivo solo es posible en las enfermedades de etiología conocida. Así la supresión del hábito tabáquico podría tener un efecto beneficioso en la resolución o estabilización de patologías como la NID, BR-EPID o histiocitosis X. La supresión de fármacos potencialmente causantes es obligada, aunque en ocasiones sea muy difícil encontrar la relación causa efecto. En las AAE, será fundamental detectar el antígeno causal para evitar su progresión, si bien esto no siempre es posible.

La supresión de la alveolitis es el único medio terapéutico en las EPID de causa desconocida. Los fármacos que se utilizan son glucocorticoides e inmunodepresores. Las indicaciones y la duración del tratamiento varían según el tipo de EPID. Cuando predominan las imágenes de reticulación y panalización que indican fibrosis y cambios irreversibles, y son el estadio final de distintas EPID, el tratamiento antiinflamatorio probablemente ya tenga muy poco valor y el manejo del paciente debiera ser paliativo y sintomático.

Hay que tener en cuenta que algunas de las EPID van a precisar de un tratamiento intenso y en ocasiones prolongado con corticoesteroides. Un especial seguimiento, y manejo médico óptimo será fundamental para patologías como la diabetes mellitus, la hipertensión arterial o la osteoporosis.

El trasplante pulmonar es la última opción terapéutica para las EPID que progresan a fibrosis y causan insuficiencia 
respiratoria. Los candidatos a trasplante deben cumplir los requisitos generales de cualquier candidato a trasplante pulmonar y no presentar contraindicaciones (Tabla 4).

\section{Una mirada específica}

\section{a la Fibrosis Pulmonar Idiopática}

Dentro de las EPID idiopáticas (NII) está la FPI. Ésta es una enfermedad diferenciada, idiopática, progresiva y fibrosante que está limitada a los pulmones. Afecta generalmente a adultos mayores de 50 años y se asocia con un patrón radiológico y/o histológico de neumonía intersticial usual (NIU). Esta enfermedad presenta en el momento actual unas características clínicas, radiológicas y patológicas bastante bien definidas. Esto ha sido posible en gran medida gracias al interés mostrado por la comunidad científica en los últimos años y las normativas y consensos realizados por distintas sociedades científicas 2,21,22.

\section{Epidemiología}

Tiene una prevalencia estimada en España que oscila entre los 13 casos por cada 100.000 habitantes en mujeres y 20 por cada 100.000 en hombres. Se cree que en España la FPI puede estar afectando a unas 70000 personas. En los últimos años, se ha observado un incremento en su incidencia. Dentro del espectro de las EPID, la FPI y la sarcoidosis son las dos más frecuentes 5,8 . La FPI representa un 50-60\% de todas las $\mathrm{NI}^{21}$. A diferencia de la sarcoidosis, la FPI suele afectar con mayor frecuencia a hombres y con una mayor edad media ${ }^{5,6,23}$. Se ha encontrando en algunos estudios una mayor probabilidad de desarrollar FPI en pacientes fumadores ${ }^{24}$.

\section{Características Clínicas y Pruebas Complementarias}

El cuadro clínico es de comienzo insidioso y se suele caracterizar por disnea de esfuerzo progresiva. Estos pacientes, muchas veces, presentan tos sin expectoración y en ocasiones de difícil control. La demora entre el inicio de la sintomatología y el diagnóstico final es variable y puede estar entre los 6 meses y los dos años ${ }^{25}$. Se auscultan estertores crepitantes en el $90 \%$ de los pacientes y se objetivan acropaquias en el $50 \%$.

Tampoco hay alteraciones de laboratorio específicas para esta enfermedad. Se puede detectar positividad en los anticuerpos antinucleares o factor reumatoide hasta en un $20 \%$ de los casos. El papel en el diagnóstico de determinados biomarcadores como KL-6, SP-A y SP-D, fibrocitos circulantes y metaloproteinasas 1 y 7 están bajo investigación en el momento actual. Ninguno de ellos de manera individual ha sido capaz de predecir con seguridad la presencia o ausencia de FPI, por lo que es posible que un panel compuesto de distintos biomarcadores pueda emplearse en el futuro con utilidad diagnóstica ${ }^{26}$.

\section{Diagnóstico}

Un consenso entre la American Thoracic Society (ATS) y la European Respiratory Society (ERS) trataron en el año 2002 de sentar las bases en el diagnóstico de la enfermedad ba-
Tabla 4. Trasplante pulmonar en pacientes con EPID

\begin{tabular}{|c|c|}
\hline & aindicaciones: \\
\hline & Oontraindicaciones absolutas \\
\hline & $\begin{array}{l}\text { Neoplasia en los últimos } 5 \text { años } \\
\text { Disfunción de un órgano vital no corregible (corazón, riñón, hígado) } \\
\text { Infección crónica extrapulmonar incurable (incluye infección crónica } \\
\text { activa por hepatitis B, C y virus de inmunodeficiencia) } \\
\text { Deformidad significativa de la caja torácica } \\
\text { Mala adherencia terapéutica } \\
\text { Trastorno psiquiátrico importante } \\
\text { Falta de soporte social } \\
\text { Adicción a tabaco, alcohol u otras drogas (abstinencia mínima } \\
\text { documentada de } 6 \text { meses) }\end{array}$ \\
\hline & Contraindicaciones relativas \\
\hline & $\begin{array}{l}\text { Edad mayor de } 65 \text { años } \\
\text { Situación clínica crítica o inestable: ventilación mecánica invasiva, } \\
\text { shock, ECM0...) } \\
\text { Limitación funcional grave que le impida participar en un programa } \\
\text { de rehabilitación pulmonar. } \\
\text { Colonización por gérmenes virulentos o altamente resistentes } \\
\text { Obesidad (IMC > 30) } \\
\text { Osteoporosis grave y sintomática }\end{array}$ \\
\hline & Criterios para referir a Unidad de Trasplantes \\
\hline & $\begin{array}{l}\text { Evidencia histológica o radiográfica de NIU, independientemente de } \\
\text { la FVC } \\
\text { Evidencia histológica de NINE fibróticas }\end{array}$ \\
\hline & riterios de Trasplante \\
\hline & $\begin{array}{l}\text { Evidencia histológica o radiológica de NIU y cualquiera de los } \\
\text { siguientes: } \\
\text { DLCO }<39 \% \\
\text { Caída } \geq 10 \% \text { de la FVC en } 6 \text { meses de seguimiento } \\
\text { Caída de la saturación de oxígeno }<88 \% \text { en test de paseo de } 6 \\
\text { minutos } \\
\text { Panalización en TCAR (score de fibrosis }>2 \text { ) }\end{array}$ \\
\hline & $\begin{array}{l}\text { Evidencia histológica de NINE y cualquiera de los siguientes: } \\
\text { DLCO }<35 \% \\
\text { Caída } \geq 10 \% \text { de la FVC } 0 \geq 15 \% \text { de la DLCO en } 6 \text { meses de } \\
\text { seguimiento }\end{array}$ \\
\hline
\end{tabular}

NIU: neumonía intersticial usual. FVC: capacidad vital forzada. NINE: neumonía intersticial no específica. DLCO: difusión de monóxido de carbono.

sándose en una serie de criterios que combinaban características clínicas, funcionales, radiológicas y patológicas². Sin embargo la falta de sensibilidad y especificidad de estos criterios unido a la alta correlación demostrada entre los hallazgos en el TCAR y la biopsia, en la última revisión del consenso estos criterios han sido eliminados y se refuerza el papel esencial de la TCAR en el diagnóstico.

Para establecer un diagnóstico definitivo de FPI se requiere: 1) la exclusión de otras entidades clínicas definidas o enfermedades parenquimatosas pulmonares difusas de causa conocida (ej. exposición ambiental u ocupacional, enfermedades del tejido conectivo, toxicidad por fármacos...), y 2) la presencia de un patrón histológico de NIU en el examen del tejido pulmonar obtenido mediante biopsia pulmonar quirúrgica, la evidencia radiológica de patrón NIU en la TCAR 0 ambos ${ }^{22}$. 
Radiología: El patrón definitorio de NIU incluye la presencia de alteraciones reticulares de predominio subpleural y basal acompañadas de áreas de panalización con o sin bronquiectasias de tracción (Figura 4). En estos casos, siempre que no existan datos inconsistentes, se puede definir como un patrón radiológico tipo NIU. Entre los hallazgos radiológicos inconsistentes estaría un predominio en campos medios 0 superiores, predominio de las lesiones a nivel peribroncovascular, abundante vidrio deslustrado o micronódulos, presencia de quistes, patrón en mosaico o datos de atrapamiento aéreo y consolidaciones segmentarias con broncograma aéreo (Tabla 5).

- Histopatología: El patrón histológico de NIU viene definido por el cumplimiento de cuatro criterios: a) evidencia de fibrosis marcada o distorsión de la arquitectura pulmonar asociada 0 no a panalización y con un predominio subpleural y paraseptal; b) presencia de lesiones parcheadas en las que se combinan áreas fibróticas con zonas de pulmón sano; c) presencia de focos fibroblásticos y d) ausencia de hallazgos histopatológicos inconsistentes con NIU. Entre las características no compatibles con patrón NIU estaría la presencia de membranas hialinas, la presencia de focos con neumonía organizada, granulomas, marcado infiltrado inflamatorio intersticial alejado de las zonas de panalización, cambios predominantemente centrados en la vía aérea o la presencia de otros hallazgos sugestivos de un diagnóstico alternativo (Tabla 6).

A pesar de unos criterios diagnósticos muy bien establecidos, tanto la sospecha diagnóstica como su confirmación final en la práctica clínica habitual presenta luces y sombras ${ }^{27}$.

El diagnóstico de FPI debe ser preciso y riguroso. La aproximación diagnóstica, basada en el algoritmo que se presenta en la Figura 5, ha de ser dinámica, integrada y multidisciplinar (clínicos, radiólogos y patólogos expertos).

\section{Historia natural y pronóstico}

La historia natural de la FPI es impredecible en el momento del diagnóstico. La supervivencia media desde el inicio de los síntomas se sitúa entre los 2 y 5 años. A pesar de la variabilidad clínica que pueden presentar los pacientes
Figura 4. Corte de TCAR característico de un paciente con fibrosis pulmonar idiopática. Se muestra un patrón reticular de localización subpleural y la presencia de múltiples quistes de pequeño tamaño que representan áreas de panalización asociada.

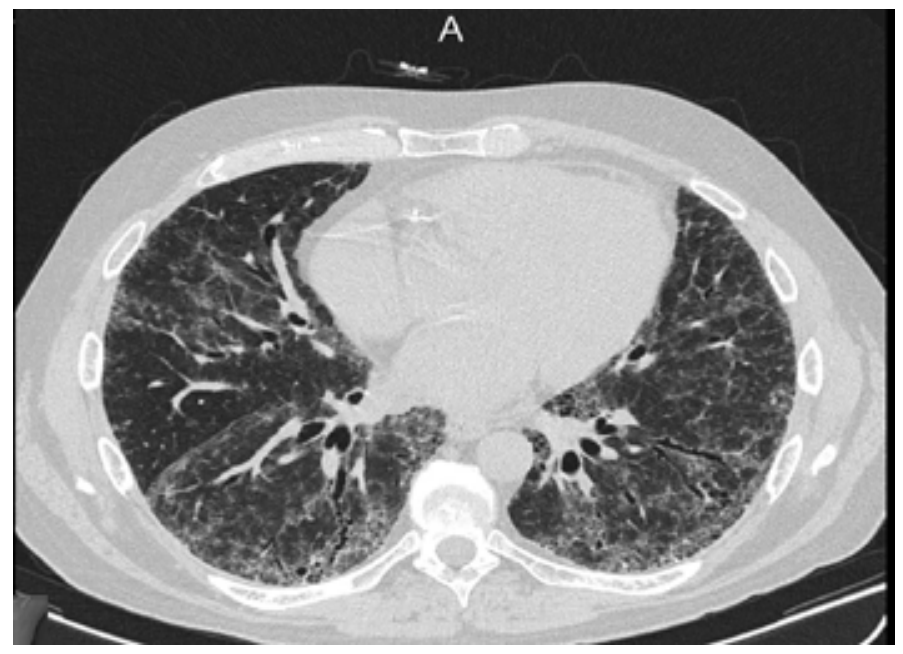

con FPI, podemos describir 3 subgrupos. Por un lado está el grupo de progresores lentos, que son aquellos que pueden tener una estabilidad inicial 0 incluso estar asintomáticos durante 2-3 años, para luego proseguir con un deterioro funcional lento pero mantenido hasta la insuficiencia respiratoria crónica. Otro grupo alternará etapas de relativa estabilidad con episodios de agudización. Existe un grupo pequeño de pacientes, que tienen una forma de progresión de la enfermedad muy aceleradaa ${ }^{28}$.

\section{Tratamiento}

La interpretación actual de la evidencia científica existente sobre el tratamiento de la FPI resulta dificultosa. Los estudios más antiguos incluían un grupo heterogéneo de enfermedades intersticiales (como la neumonía intersticial descamativa o la neumonía intersticial no específica) con una historia natural diferente a la FPI tanto en la evolución como en la respuesta al tratamiento esteroideo. Además existen muy pocos ensayos clínicos randomizados y controlados con placebo con el suficiente peso científico que permita establecer recomendaciones definitivas.
Tabla 5. Criterios de patrón de NIU en la tomografía computerizada de alta resolución (modificado de Raghu²)

\begin{tabular}{|c|c|c|}
\hline $\begin{array}{l}\text { Patrón de NIU } \\
\text { (Las cuatro } \\
\text { caracteristicas) }\end{array}$ & $\begin{array}{c}\text { POSIBLE patrón de } \\
\text { NIU (Las tres } \\
\text { caracteristicas) }\end{array}$ & $\begin{array}{c}\text { No concordante con Patrón } \\
\text { de NIU } \\
\text { (cualquiera de las siete } \\
\text { caracteristicas) }\end{array}$ \\
\hline $\begin{array}{l}\text { - Predominio } \\
\text { basal, subpleural } \\
\text { - Anomalia } \\
\text { reticular }\end{array}$ & $\begin{array}{l}\text { - Predominio basal, } \\
\text { subpleural } \\
\text { - Anomalia reticular }\end{array}$ & 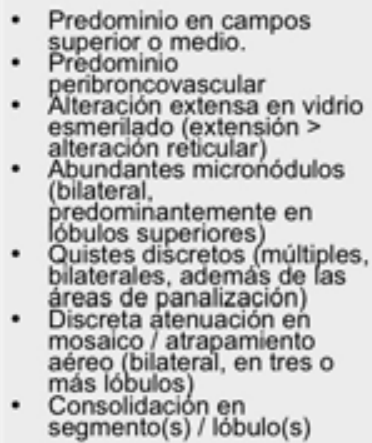 \\
\hline
\end{tabular}

Tabla 6. Patrón de NIU: criterios histopatológicos (modificado de Raghu²)

\begin{tabular}{|c|c|c|c|}
\hline $\begin{array}{l}\text { Patrón de NIU } \\
\text { (Los cuatro criterios) }\end{array}$ & $\begin{array}{l}\text { Patrón de NIU } \\
\text { PROBABLLE }\end{array}$ & $\begin{array}{l}\text { Patrón de NIU } \\
\text { POSSiBLE } \\
\text { (Los tres criberios) }\end{array}$ & $\begin{array}{l}\text { NO patrón de NIU } \\
\text { (Aguno do los sels } \\
\text { Criterios) }\end{array}$ \\
\hline $\begin{array}{l}\text { Evidencia de marcada } \\
\text { fibross / deformacisn } \\
\text { de la arquitectura, } * \text {. } \\
\text { panalzación con } \\
\text { distribucion } \\
\text { predominantemente } \\
\text { subpleuralivaraseptal } \\
\text { Afectacion parcheada } \\
\text { del parénquima } \\
\text { pulmonar por fibrosis } \\
\text { Presencia de focos } \\
\text { fibrobiasticos } \\
\text { Ausencia de } \\
\text { caracteristicas } \\
\text { incompatibles con } \\
\text { diagnsstico de NIU } \\
\text { guo sugieran un } \\
\text { diagnjestico alternasivo. }\end{array}$ & 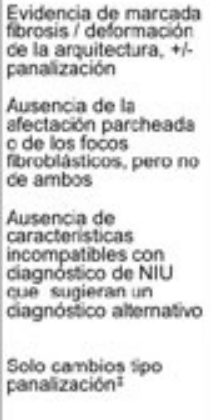 & 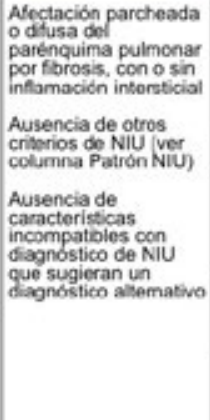 & $\begin{array}{l}\text { - Mombranas hialinas } \\
\text { - Neumonia } \\
\text { organizativa } \\
\text { - Granulomas } \\
\text { - Marcado infiltrado } \\
\text { celular inflamatorio } \\
\text { intersticial aderás } \\
\text { de la panalizacon } \\
\text { - Cambios centrados } \\
\text { pradominantes en } \\
\text { - as vias aéreas } \\
\text { - Oras caracteristicas } \\
\text { sugestivas de in } \\
\text { diagnostco } \\
\text { aliernativo. }\end{array}$ \\
\hline
\end{tabular}

NIU: neumonía intersticial usual 
Figura 5. Algoritmo diagnóstico de la Fibrosis Pulmonar Idiopática (modificado de ${ }^{27}$ )

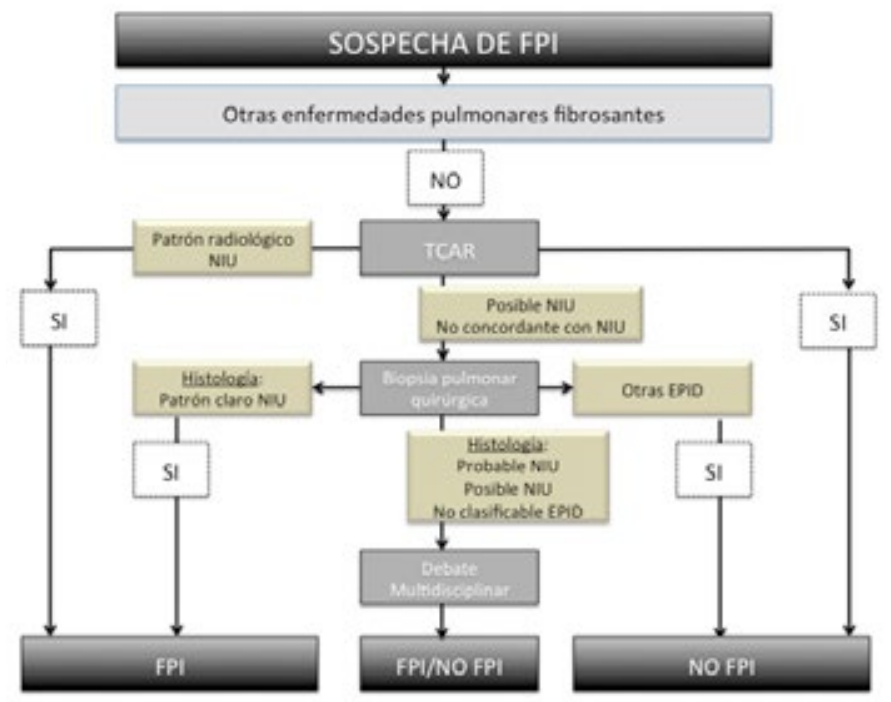

A día de hoy el único tratamiento que aumenta la supervivencia en los pacientes con FPI es el trasplante de pulmón en casos seleccionados.

- Tratamiento clásico: corticoides e inmunosupresores. A pesar de que no se había llevado a cabo ningún estudio prospectivo, controlado con placebo con la asociación de corticoides e inmunosupresores (azatioprina o ciclofosfamida) en el tratamiento de la FPI, estos se emplearon ampliamente siguiendo las recomendaciones internacionales elaboradas por la ATS y ERS en el año 2000. En el momento actual y con la evidencia científica que tenemos, podemos decir que a) Ios corticoides NO han demostrado ninguna eficacia en el tratamiento de la FPI ${ }^{29}$; b) la asociación de corticoides, azatioprina y $\mathrm{N}$-acetil cisteína no solo NO ha demostrado ninguna eficacia en el tratamiento de la FPI, sino que su uso incluso podría tener efectos fatales sobre el paciente ${ }^{30}$. Por todo ello, y en base a las últimas recomendaciones no está recomendado su uso ${ }^{22}$.

- Posibilidades actuales:

- N-Acetilcisteina (NAC): Dado su efecto antioxidante, su relativo escaso coste y buen perfil de efectos secundarios, la NAC se está empleando en el tratamiento de la FPI. La dosis empleada es de $600 \mathrm{mg} / 8 \mathrm{~h}$. Hasta que se conozcan los resultados del estudio PANTHER no se podrá dilucidar la verdadera eficacia de la NAC en el tratamiento de la FPl30.

- Pirfenidona: La pirfenidona es un fármaco con propiedades antiinflamatorias y antifibróticas de administración oral. Su eficacia fue testada en 3 ensayos clínicos fase III, uno en población asiática y los dos restantes, publicados conjuntamente (Capacity 004 y 006) ${ }^{31}$, como estudios multicéntricos que incluyeron población europea y americana. Mientras que en uno de ellos (004) alcanzó el objetivo primario definido por la tasa de caída en el FVC, en el segundo (006) no se demostró dicho efecto, debido a que los pacientes del grupo control no se comportaron, en cuanto a su pérdida de CV, como se esperaba por la experiencia comprobada en otros pacientes controles de FPI. Sin embargo un meta-análisis ${ }^{32}$ de los objetivos primarios de los estudios publicados demostró que piffenidona reduce de manera significativa la tasa de caída de la FVC comparado con placebo. También se observaron efectos beneficiosos en varios objetivos secundarios como el tiempo de progresión libre de enfermedad, cambio categórico de la FVC o distancia recorrida en el test de paseo. En base a los estudios publicados estaría indicada en el tratamiento de la FPI de intensidad leve-moderada definida por FVC $>50 \%$, DLCO $>35 \%$ y una distancia caminada en el test de paseo de 6 minutos mayor a 150 metros. En el momento actual es un fármaco que si bien ha sido aprobado por la Agencia Europea del Medicamento (EMA) en 2011, aún no se ha comercializado en España y que en Estados Unidos la Food and Drug Administration (FDA) ha requerido la realización de un ensayo clínico extra que aclare su eficacia para que sea aprobado.

- Trimetropim/sulfametoxazol: recientemente se ha publicado un ensayo clínico en el que en un grupo de 181 pacientes con FPI fueron tratados con cotrimoxazol 0 placebo; observaron que la adicción de cotrimoxazol al tratamiento estándar no tuvo efecto sobre la función pulmonar pero sí tuvieron una mejoría en la calidad de vida y una reducción en la mortalidad por todas las causas en aquellos que tomaron el tratamiento ${ }^{33}$.

- Tratamientos no farmacológicos:

- Trasplante pulmonar: El trasplante de pulmón es actualmente el único tratamiento que ha demostrado prolongar la supervivencia y mejorar la calidad de vida de los pacientes con FPI. Desgraciadamente, el número de pacientes que se puede beneficiar de esta técnica es limitado (menores de 65 años y que cumplan los criterios requeridos - Tabla 4). Estos pacientes presentan una alta mortalidad en la lista de espera, lo que hace imprescindible que sean remitidos a un centro trasplantador de forma precoz, generalmente en el momento del diagnóstico.

- Oxigenoterapia domiciliaria: La indicación de oxigenoterapia en pacientes con FPI e insuficiencia respiratoria es fruto de la extrapolación de los estudios realizados en pacientes con EPOC. Hoy en día se acepta recomendar administrar oxigenoterapia crónica domiciliaria ante la constatación de hipoxemia significativa en reposo ( $\mathrm{SaO2}$ $<88 \%$ ) 0 caída en la saturación de oxígeno importante durante el ejercicio.

- Rehabilitación respiratoria: No existen estudios que hayan demostrado los efectos beneficiosos de la rehabilitación sobre la supervivencia a largo plazo, sin embargo parece tener un impacto beneficioso en la distancia caminada en la prueba de 6 minutos y en la calidad de vida ${ }^{34}$.

\section{Conclusiones}

Existen más de 150 entidades que puedan provocar EPID y suelen compartir características clínicas, radiológicas o funcionales. Una exhaustiva historia clínica, prestando especial 
atención a exposiciones y datos exploratorios de secundarismos será fundamental para una correcta aproximación diagnóstica. El diagnóstico de las EPID debe ser secuencial, dinámico y multidisciplinar. El tratamiento será individualizado a cada EPID, y se basará en evitar la exposición al potencial agente causal cuando exista y tratamientos antiinflamatorios en aquellos casos que no hayan evolucionado a fibrosis franca y que se prevea algún beneficio.

La FPI tiene unas manifestaciones clínico-radiológicas bien definidas y se caracteriza por la presencia de un patrón histológico de neumonía intersticial usual (NIU) en una muestra de parénquima pulmonar. En determinados casos el diagnóstico puede basarse en criterios exclusivamente clínicoradiológicos. En la actualidad no existe ningún tratamiento farmacológico que modifique el pronóstico de la enfermedad. A los pacientes que cumplan los criterios requeridos para un trasplante de pulmón se les remitirá de forma precoz a una unidad de trasplante pulmonar.

\section{Bibliografía}

1. Xaubet A, Ancochea J, Blanquer R, Montero C, Morell F, Rodriguez Becerra E, et al. Diagnosis and treatment of diffuse interstitial lung diseases. Archivos de bronconeumología 2003;39:580-600.

2. American Thoracic Society/European Respiratory Society. International Multidisciplinary Consensus Classification of the Idiopathic Interstitial Pneumonias. Am J Respir Crit Care Med 2002; 165: 277-304.

3. Gulati M. Diagnostic assessment of patients with interstitial lung disease. Prim Care Respir J 2011;20:120.

4. Coultas DB, Zumwalt RE, Black WC, Sobonya RE. The epidemiology of interstitial lung diseases. Am. J. Respir. Crit. Care Med. 1994;150:967-72.

5. Xaubet A, Ancochea J, Morell F, Rodriguez-Arias JM, Villena V, Blanquer R, et al. Report on the incidence of interstitial lung diseases in Spain. Sarcoidosis Vasc Diffuse Lung Dis 2004;21:64-70.

6. Karakatsani A, Papakosta D, Rapti A, Antoniou KM, Dimadi M, Markopoulou A, et al. Epidemiology of interstitial lung diseases in Greece. Respir Med 2009;103:1122-9.

7. Flaherty KR, Travis WD, Colby TV, Toews GB, Kazerooni EA, Gross BH, et al. Histopathologic variability in usual and nonspecific interstitial pneumonias. Am. J. Respir. Crit. Care Med. 2001;164:1722-7.

8. Morell F, Levy G, Orriols R, Ferrer J, De Gracia J, Sampol G. Delayed cutaneous hypersensitivity tests and lymphopenia as activity markers in sarcoidosis. Chest 2002;121:1239-44.

9. Meyer KC, Raghu G, Baughman RP, Brown KK, Costabel U, Bois du RM, et al. An official American Thoracic Society clinical practice guideline: the clinical utility of bronchoalveolar lavage cellular analysis in interstitial lung disease. Am J Resp Crit Care Med 2012;185:1004-14.

10. Lama VN, Martinez FJ. Resting and exercise physiology in interstitial lung diseases. Clin. Chest Med. 2004;25:435-53-V.

11. Chetta $A$, Marangio $E$, Olivieri D. Pulmonary function testing in interstitial lung diseases. Respiration 2004;71:209-13.

12. Chetta A, Aiello M, Foresi A, Marangio E, D'Ippolito R, Castagnaro A, et al. Relationship between outcome measures of six-minute walk test and baseline lung function in patients with interstitial lung disease. Sarcoidosis Vasc Diffuse Lung Dis 2001;18:170-5.

13. Ohshimo S, Bonella F, Cui A, Beume M, Kohno N, Guzman J, et al. Significance of bronchoalveolar lavage for the diagnosis of idiopathic pulmonary fibrosis. American Journal of Respiratory and Critical Care Medicine 2009;179:1043-7.
14. Roggli VL, Gibbs AR, Attanoos R, Churg A, Popper H, Cagle P, et al. Pathology of asbestosis- An update of the diagnostic criteria: Report of the asbestosis committee of the college of american pathologists and pulmonary pathology society. Arch. Pathol. Lab. Med. 2010;134:462-80.

15. Dooms C, Seijo L, Gasparini S, Trisolini R, Ninane V, Tournoy KG. Diagnostic bronchoscopy: state of the art. European Respiratory Review 2010;19:229-36.

16. Leslie KO. Historical perspective: a pathologic approach to the classification of idiopathic interstitial pneumonias. Chest 2005;128:513S-519S.

17. Pajares V, Torrego A, Puzo C, Lerma E, Gil De Bernabé MA, Franquet T. Transbronchial lung biopsy using cryoprobes. Archivos de bronconeumología 2010;46:111-5.

18. Babiak A, Hetzel J, Krishna G, Fritz P, Moeller P, Balli T, et al. Transbronchial cryobiopsy: a new tool for lung biopsies. Respiration 2009;78:203-8.

19. Rocco G, Martin-Ucar A, Passera E. Uniportal VATS wedge pulmonary resections. Ann. Thorac. Surg. 2004;77:726-8.

20. Fibla JJ, Molins L, Blanco A, Royo I, Martínez Vallina P, Martínez N, et al. Videoassisted thoracoscopic lung biopsy in the diagnosis of interstitial lung disease: a prospective, multi-center study in 224 patients. Archivos de bronconeumología 2012;48:81-5.

21. Ancochea J, Gómez J, Villar J, Xaubet A, Committee OBOTSSS. Consensus on the diagnosis of idiopathic interstitial pneumonia. Archivos de bronconeumología 2010;46:1-21.

22. Raghu G, Collard HR, Egan JJ, Martinez FJ, Behr J, Brown KK, et al. An Official ATS/ ERS/JRS/ALAT Statement: Idiopathic Pulmonary Fibrosis: Evidence-based Guidelines for Diagnosis and Management. American Journal of Respiratory and Critical Care Medicine 2011;183:788-824.

23. Fernández Pérez ER, Daniels CE, Schroeder DR, St Sauver J, Hartman TE, Bartholmai BJ, et al. Incidence, prevalence, and clinical course of idiopathic pulmonary fibrosis: a population-based study. Chest 2010;137:129-37.

24. Baumgartner KB, Samet JM, Stidley CA, Colby TV, Waldron JA. Cigarette smoking: a risk factor for idiopathic pulmonary fibrosis. Am. J. Respir. Crit. Care Med. 1997;155:242-8.

25. Kim DS, Collard HR, King TEJ. Classification and natural history of the idiopathic interstitial pneumonias. Proc 2006;3:285-92.

26. van den Blink B, Wijsenbeek MS, Hoogsteden HC. Serum biomarkers in idiopathic pulmonary fibrosis. Pulm Pharmacol Ther 2010;23:515-20.

27. Marcos PJ, Valenzuela C, Ancochea J. De la exclusión a la certidumbre. El recorrido hacia el diagnóstico de la fibrosis pulmonar idiopática. Archivos de bronconeumología 2012;48:7-12.

28. Selman M, Carrillo G, Estrada A, Mejia M, Becerril C, Cisneros J, et al. Accelerated variant of idiopathic pulmonary fibrosis: clinical behavior and gene expression pattern. PLOS ONE 2007;2:e482.

29. Richeldi L, Davies HR, Ferrara G, Franco F. Corticosteroids for idiopathic pulmonary fibrosis. Cochrane Database Syst Rev 2003;:CD002880.

30. Idiopathic Pulmonary Fibrosis Clinical Research Network, Raghu G, Anstrom KJ, King TE, Lasky JA, Martinez FJ. Prednisone, azathioprine, and N-acetylcysteine for pulmonary fibrosis. N Engl J Med 2012;366:1968-77.

31. Noble PW, Albera C, Bradford WZ, Costabel U, Glassberg MK, Kardatzke D, et al. Pirfenidone in patients with idiopathic pulmonary fibrosis (CAPACITY): two randomised trials. Lancet 2011;377:1760-9.

32. Cottin V. Changing the idiopathic pulmonary fibrosis treatment approach and improving patient outcomes. European Respiratory Review 2012;21:161-7.

33. Shulgina L, Cahn AP, Chilvers ER, Parfrey H, Clark AB, Wilson ECF, et al. Treating idiopathic pulmonary fibrosis with the addition of co-trimoxazole: a randomised controlled trial. Thorax 2013;68:155-62.

34. Holland A, Hill C. Physical training for interstitial lung disease. Cochrane Database Syst Rev 2008;:CD006322.

35. ATS Committee on Proficiency Standards for Clinical Pulmonary Function Laboratories. ATS statement: guidelines for the six-minute walk test. Am J Respir Crit Care Med 2002;166:111-7. 